\title{
Signal response amplification of scale-free networks
}

\author{
LIU ZongHua \\ Institute of Theoretical Physics and Department of Physics, East China Normal University, Shanghai 200062, China
}

Received March 26, 2011; accepted May 9, 2011

\begin{abstract}
In many animals and insects, hearing is very acute to the faintest of sounds; the underlying mechanism can be explained by self-tuning. Recently, signal response amplification has been shown to be implemented through networks exhibiting scale-free topology, which has potential applications in artificial information processing systems and devices. We review in this paper the main results obtained in networked double-well oscillators and briefly discuss future research directions.
\end{abstract}

signal response, scale-free network, self-tuning, double-well oscillator

Citation: $\quad$ Liu Z H. Signal response amplification of scale-free networks. Chinese Sci Bull, 2011, 56: 3623-3629, doi: 10.1007/s11434-011-4701-y

Systems that can detect and amplify signals at specific frequencies are commonplace in the natural world and most notably in the visual and auditory systems of animals [1]. Signal detection in animals is through light- and auralsensitive organs, constituted by a large number of networked units. For example, cells in living organisms respond to their environment by an interconnected network of receptors, messengers, protein kinases and other signaling molecules [2-4]. One of the more prominent features of our hearing system is the ability to perceive sound stimuli that range over six orders of magnitude in sound pressure [5]. Hair cells within the cochlear are stimulated by sound waves, the induced motion being amplified at characteristic locations that depends on the frequencies of sound. These cells transmit signals to the auditory nerve [6].

It is well known that many animals and insects have the ability to detect faint sounds from their environment. Physiological evidence exists for a range of animals and insect auditory systems that this active audition is due to Hopf bifurcations [7-9]. Models have also been proposed to develop the underlying mechanism behind enhanced amplification in hearing systems, i.e. self-tuned critical oscillations of hair cells nearby the Hopf bifurcation. For example, Camalet et al. argued that the active amplification of faint

email: zhliu@phy.ecnu.edu.cn sounds is provided by a dynamic system maintained at the threshold of an oscillatory instability [2]. Stoop et al. suggested a van-der-Pol oscillator model to explain the active signal amplification in the Drosophila hearing system [4]. McCullen et al. claimed that the generalized van-der-Pol oscillator with a 3-cell feed-forward network can explain the signal amplification well [1]. In detail, a double-well potential may be used to show how self-tuning works [10]. This potential can be expressed in the form $U(x)=a(x-1)^{2}$ $\times(x+2)^{2}$, where $a$ controls the barrier height of the potential; $x= \pm 1$ are the locations of the two minima. Suppose an oscillator has probability $w_{+}\left(w_{-}\right)$of jumping from the right (left) well to the left (right) well; then self-tuning means that the parameter $a$ can be self-adjusted by the following equation:

$$
\frac{\mathrm{d} a(t)}{\mathrm{d} t}=-\alpha a(t)+\beta\left[w_{+}(t) p_{+}(t)+w_{-}(t) p_{-}(t)\right],
$$

where $p_{+}\left(p_{-}\right)$denotes the probability that the oscillator stays at $x= \pm 1$. If $w_{+}$and $w_{-}$are small, the first term in eq. (1) will be larger than the second term and thus result in a decrease in $a$. For larger $w_{+}$and $w_{-}$, the first term in eq. (1) will be smaller than the second term and thus result in an increase in $a$. Therefore, the parameter $a$ is self-tuned to an optimal value by switching probabilities $w_{+}$and $w_{-}$.

Scientists and engineers frequently take inspiration from 
nature, although signal detection is one area where biology has excelled in producing systems with superior characteristics over man-made devices. Artificial information-processing systems and devices usually consist of many nonlinear oscillators [11-13]. An interesting question is what can be incorporated into artificial systems and devices from these self-tuning auditory systems of animals and insects. Most of the technical and biology networks have been found to be scale-free (SF) networks where node degrees satisfy a power-law distribution [14,15]. Accruing evidence suggests that the functional connections between different areas of human and cat brains are also SF networks [16-18]. Recently, the brain, a vastly interconnected network, has been shown to behave much like the internet [19]. Thus, understanding how the SF topology of networked units influences signal detection abilities is an essential task.

This topic has been intensely studied recently [20-23]. By considering a SF network with a double well unit on each node, the network topology has been found to have a strong effect on the signal response, especially for weak external signals. We summarize three important achievements: (1) Acebron et al. first considered weak signaling for a Barabasi-Albert (BA) SF network and found that the hub has the ability to amplify weak signals in a middle range of coupling strength [20]. (2) Liu et al. discovered that when adding noise, the hub will exhibit a double resonance associated with noise and coupling strengths [21]. (3) Kondo et al. showed that the hub and other nodes also contribute to signal amplification, i.e. each fifty-fifty unit corresponds to a maximum amplification at a specific coupling strength [23]. In this paper, we will review these results and briefly discuss future research directions.

\section{Signal amplification on the hub}

In accordance with the BA algorithm, we first construct a SF network with total node number $N$, average links $\langle k\rangle$, and degree distribution $p(k) \sim k^{-3}[24,25]$. Next, on each node we set a double-well oscillator linked with others, each link having coupling strength $\lambda$. Interactions among the oscillators can only occur via these links. The networked doublewell systems are as follows:

$$
\dot{x}_{i}=-\mathrm{d} V\left(x_{i}\right) / \mathrm{d} x_{i}+\sum_{j=1}^{k_{i}} \lambda\left(x_{j}-x_{i}\right)+A \cos \omega t
$$

where the potential $V(x)=(x-1)^{2}(x+1)^{2}, k_{i}$ denotes the number of links of node $i$, and $A \cos \omega t$ the weak signal. This potential has an unstable maximum at $x=0$ and two minima at $x= \pm 1$. Without couplings, an oscillator will ultimately stay at one of the two minima. We choose a signal strength $A=0.8$ so that there is no jumping at non-hub nodes. In this paper, we fix $N=500,\langle k\rangle=6$ and $\omega=0.5$ for the SF network if without specific illustration. Initially, $\left\{x_{i}(t=0)\right\}$ $(i=1,2, \cdots, N)$ are randomly chosen to be either 1 or -1 with probability $1 / 2$. Figure 1 shows the stationary state of two typical nodes; the dotted and solid lines represent the evolutions on the hub with links $k_{\text {hub }}=68$ and a general node with links $k_{\mathrm{ge}}=5$, respectively, and (a), (b) and (c) correspond to coupling strengths $\lambda=0.02,0.05$ and 0.1 , respectively. From Figure 1(a) we see that the general node oscillates around the equilibrium point $x=1$ whereas the hub oscillation is a little away from $x=1$ and with much larger amplitude than that of the general node. At the optimal coupling strength $\lambda=0.05$, the hub will oscillate between $x=1$ and $x=-1$, i.e. around $x=0$; see Figure 1(b). If $\lambda$ is greater than the optimal value, the hub oscillation will be away from $x=0$, again because of the synchronization of oscillators; see Figure 1(c). That is, the hub oscillation is significantly amplified in a mid-range in the coupling strength whereas the general node is not.

To characterize the signal response amplification at the hub, Acebron et al. introduced a gain $G$ as a measure of the information processing efficiency and find that the hub has the ability to amplify weak signals in a mid-range in the coupling strength [20]. Specifically, $G$ is defined by

$$
G \equiv \max _{i} a_{i} / A \equiv a_{L} / A,
$$

where $a_{i} \equiv\left[\max _{t} x_{i}(t)-\min _{t} x_{i}(t)\right] / 2$ determines the oscillation amplitude for the $i$-th node and $a_{L}$ the maximal amplitude given by the $L$-th node. Although $G$ depends on the maximal $a_{i}$ which is not necessary for the hub, both the theoretical analysis and numerical simulations in the work of Acebron et al. [20] focus on the hub. Implicitly, they assume that the maximum response occurs on the hub, which is correct only for a very weak signal. Figure 2 shows how the gain on the hub changes with coupling strength $\lambda$ where the "squares", "circles" and "triangles" identify results for signal strengths $A=0.4,0.8$ and 1.2 , respectively. Note that $G$ is significantly amplified in a range around $\lambda=0.05$.
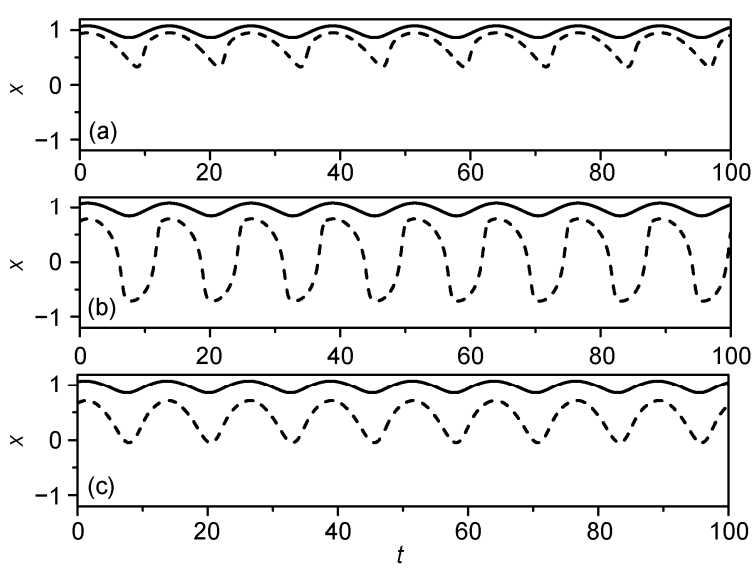

Figure 1 Signal response on a BA network with $N=500$, with coupling strength, corresponding to panels $\lambda=0.02$ (a), $\lambda=0.05$ (b) and $\lambda=0.1$ (c). The dotted and solid lines represent the dynamics of the hub with links $k_{\text {hub }}=68$ and of a general node with links $k_{\mathrm{ge}}=5$, respectively. 


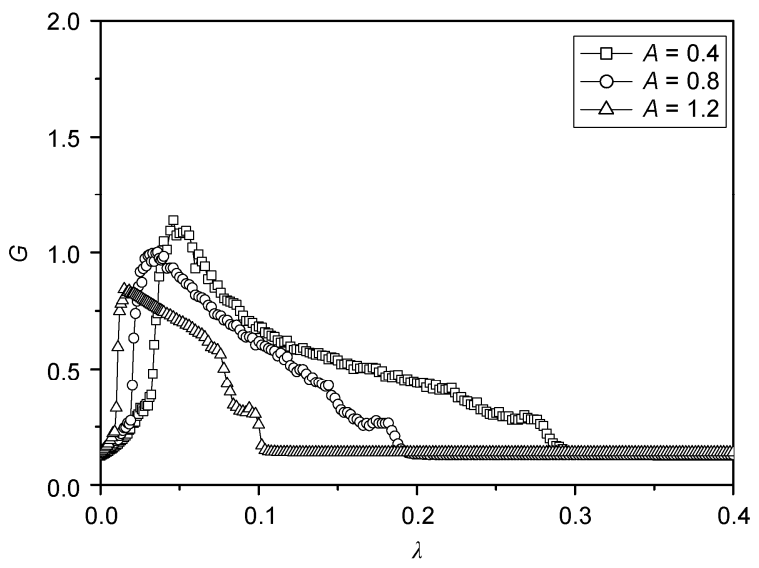

Figure 2 The gain $G$ of the hub as a function of coupling strength $\lambda$ for a SF network with $N=500$ and $\langle k\rangle=6$; "squares", "circles" and "triangles" mark signal strengths $A=0.4,0.8$ and 1.2 , respectively.

Considering that the hub has a large degree, Acebron et al. treated the hub and its neighbors as a starlike network. That model explained the observed behaviors in Figure 2 very well; see details in [20].

\section{Double stochastic resonance}

Stochastic resonance (SR) was originally investigated by Benzi et al. to model switching of the Earth's climate between ice ages and periods of relative warmth with a period of about $10^{5}$ years [26]. An important ingredient of SR is the existence of optimal noise strength where the signal-tonoise ratio (SNR) of a nonlinear system is found to be sensitive to the noise amplitude and can reach a maximum value at an optimal noise level [27-29]. A large number of applications of SR have been reported [11-13,27-32], especially in biological systems. One new aspect here is that we have an extra coupling strength parameter; detectability determined from eq. (2) turns out to attain the maximum values at finite coupling strength. Thus, we may expect a type of double resonance with respect to noise strength (as in SR) and coupling strength [21].

In a second application, signal detection in cells or neurons is modeled through the jumping/firing between the two minima of the potential $V(x)$ but not the oscillations around one of the two minima. Liu et al. added noise to eq. (2) to induce and sustain the jumping behavior [21]. Eq. (2) then becomes

$$
\dot{x}_{i}=-\mathrm{d} V\left(x_{i}\right) / \mathrm{d} x_{i}+\sum_{j=1}^{k_{i}} \lambda\left(x_{j}-x_{i}\right)+A \cos \omega t+\xi_{i}(t),
$$

where noise $\xi_{i}(t)$ is treated as independent Gaussian white noise with average zero satisfying

$$
\left\langle\xi_{i}(t) \xi_{j}\left(t^{\prime}\right)\right\rangle=2 T \delta_{i j} \delta\left(t-t^{\prime}\right) .
$$

With noise, the dynamics of eq. (4) will be completely different from that of eq. (2) as noise may induce jumping/ firing between the two equilibrium positions $x= \pm 1$. Figure 3 shows the results for $T=0.2$ with panels (a), (b) and (c) corresponding to $\lambda=0.02,0.05$ and 0.1 , respectively; the solid lines in each panel oscillate between the two equilibrium positions, in contrast to those in the panels of Figure 1 for $T=0$ where these lines oscillate around $x=1$. Without noise, no jumps occur and thus the system remains near its initial condition; noise induces jumping and thus diverges from its initial condition. We can say that the former has a form of memory, the latter a loss of memory, for its initial state.

From Figure 3, we conclude that the maximum $a_{i}$ of all the nodes will become approximately the same and will not change with coupling strength. Thus, $G$ is no longer a good quantity to characterize the influence of noise and coupling. Liu et al. suggested that in this situation, a good quantity to characterize the influence of noise and coupling is the SNR; i.e. how the coupling strength and noise strength influence the SNR [20]. To obtain the SNR, we first fix $T$ and let the coupling strength $\lambda$ change. By taking a time series for each $\lambda$ and evaluating the Fourier transformation to obtain its power spectrum, we can calculate the SNR at reference frequency $\omega[12,13]$. Figure 4(a) and (b) show how the variation of SNR with coupling strength at $T=0.2$ for the general node and the hub, respectively. The general node and the hub show stochastic resonance dependent on coupling strength, but the hub is about 20 times larger than the general node indicating the hub amplifies weak signals detected by a large number of the surrounding general nodes.

Next, we fix $\lambda$ while varying $T$. We find that the SNR also shows resonance dependent on noise strength $T$ similar to traditional SR. Figure 4(c) and (d) show the results for the general node and the hub, respectively. In summary, SF networked two-state systems show an interesting double SR at the hub, i.e. resonance for both coupling strength and
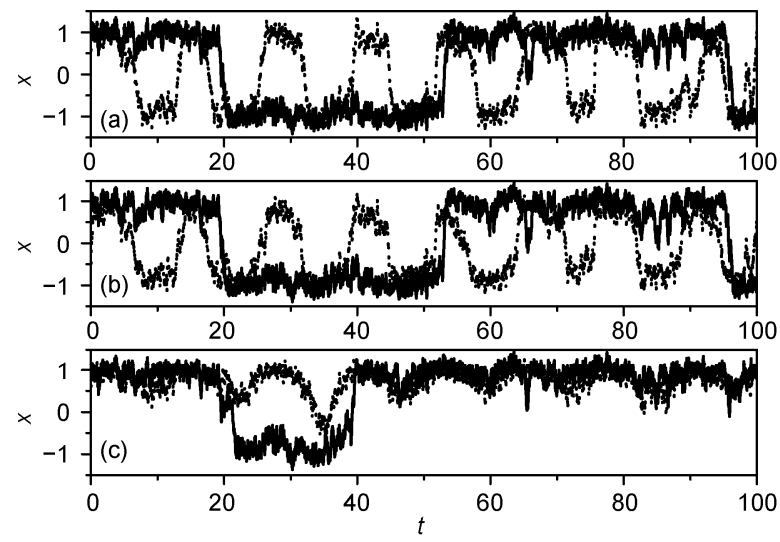

Figure 3 Influence of noise on the dynamics of double-well systems for a BA network with $N=500$ and $T=0.2$ with varying coupling strength $\lambda=0.02$, 0.05 and 0.1 corresponding to panels (a), (b) and (c), respectively; the dotted and solid lines correspond to the hub with links $k_{\text {hub }}=68$ and a general node with links $k_{\mathrm{ge}}=5$, respectively. 

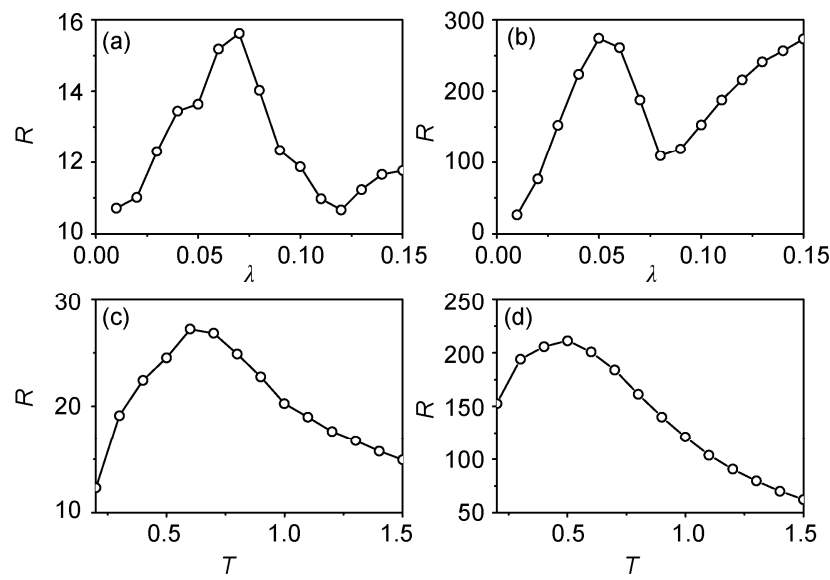

Figure 4 Variation in SNR with coupling strength and noise strength for BA networked oscillators with $N=500$. In (a) and (b) $T=0.2$, and in (c) and (d) $\lambda=0.03$. (a) and (c) correspond to results for a general node with links $k_{\mathrm{ge}}=5$; (b) and (d) that for the hub with links $k_{\text {hub }}=68$.

noise strength. Its theoretical explanation can be found in [21].

\section{Contribution of non-hub nodes to the signal amplification}

Although Acebron et al. have showed that the existence of the hub unit played a decisive role in increasing gain, their theory is only limited to the hub or when the hub jumps between the two minima. As a result, the properties of a SF network are not fully elucidated, and the roles of network structures such as the power-law degree distribution [14] remain to be clarified. To illustrate the function of non-hub nodes, Kondo et al. developed a one-body theory that enables analytic expressions to be obtained for the gain $G$ and the degree $k_{L}$ of the unit, as well as the maximum response to an input signal in terms of the coupling strength $\lambda$ [23].

Reconsidering the model of eq. (2), let us first introduce the concept of a fifty-fifty unit. A unit $i$ belongs to the fiftyfifty unit $F$ if about half of its neighbors $\left(k_{i} / 2\right)$ are oscillating in the left well $(x \approx-1)$ and the rest in the right well $(x \approx 1)$ in a stationary state; we express this as $i \in F$. The main observation, which enabled us to reduce eq. (2) to a one-body problem for $G$, is that the unit $L$, which realizes maximum response $a_{L}$, belongs to the fifty-fifty unit, i.e. $L \in F$. This first occurred to us through data analysis of our numerical experiments that later turned out to be reasonable.

If $i \in F$ and is located on the left well at some time, the unit $i$ feels an attractive force from $k_{i} / 2$ units in the right well with nearly no force from the $k_{i} / 2$ units in the left well. If the force happens to be strong enough to pull the unit $i$ to the right well over the potential barrier of $V(x)$, it moves to the right well and a similar process occurs to bring the unit $i$ back to the left well. This to-and-fro motion is the mechanism needed for a unit to perform large amplitude oscilla- tion around the center $x=0$.

This is easily formulated using eq. (2) as follows: If we choose arbitrarily a unit $i \in F$, to be labeled by $f$ for convenience, we can write down eq. (2) as

$$
\begin{aligned}
\dot{x}_{f} & =-4 x_{f}^{3}+\left(4-\lambda k_{f}\right) x_{f}+A \sin (\omega t) \\
& \equiv-\mathrm{d} V_{\text {eff }}\left(x_{f}\right) / \mathrm{d} x_{f}+A \sin (\omega t),
\end{aligned}
$$

where the assumption $\sum_{j=1}^{k_{i}} x_{j} \ll \sum_{j=1}^{k_{i}} x_{f}=k_{f} x_{f}$ is used. In a stationary state $x_{f}(t)$ performs a periodic oscillation with period $\tau_{p} \equiv 2 \pi / \omega$ and we denote the solution as $x_{f, p}(t)$. The maximum value of $x_{f}$ can be obtained by putting $x_{f}=0$ in eq. (6). Let $A_{\max }$ be the value of $A \sin (\omega t)$ and let $g\left(x_{f, \text { max }}\right)$ be $-\mathrm{d} V_{\text {eff }}\left(x_{f, \text { max }}\right) / \mathrm{d} x_{f, \text { max }}$ when $x_{f, p}(t)$ takes the maximum value $x_{f, \text { max }}$. The pair $\left(x_{f, \max }, A_{\max }\right)$ is easily seen to satisfy the following algebraic equation:

$$
g\left(x_{f, \max }\right) \equiv-\mathrm{d} V_{\text {eff }}\left(x_{f, \text { max }}\right) / \mathrm{d} x_{f, \text { max }}=-A_{\text {max }} .
$$

Kondo et al. proved that in general, we have [21]

$$
A_{\max } \approx A, A_{\min } \approx-A \text {. }
$$

Thus eq. (7) becomes

$$
g\left(X_{f}\right) \equiv-4 X_{f}^{3}-\left(\lambda k_{f}-4\right) X_{f}= \pm A .
$$

Figure 5 shows graphically how the amplitude of oscillation changes with $\lambda k_{f}$ for $A=0.8$, where the solid curve represents $y=g(X)$, the dashed lines $y= \pm A$, the bold arrow the oscillation amplitude, and (a), (b), (c) and (d) correspond to cases $\lambda k_{f}=0,4-3 A^{2 / 3}, 1.8$ and 3.6, respectively; here $4-3 A^{2 / 3}$ represents a critical value for $\lambda k_{f}^{\mathrm{c}}$, the derivation of which will be given below in eq. (11).
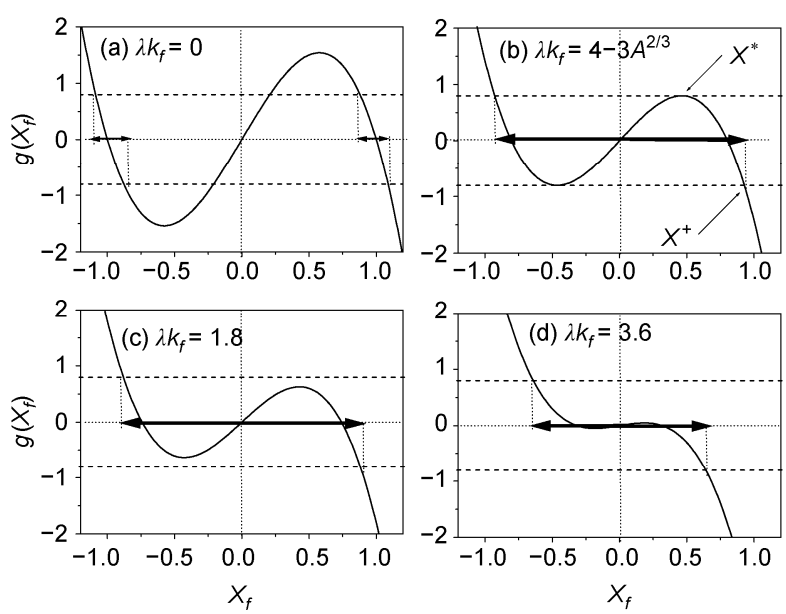

Figure 5 Variation in the oscillation amplitude with parameter $\lambda k_{f}$ setting $A=0.8$ in eq. (9). (a), (b), (c) and (d) correspond to $\lambda k_{f}=0,4-3 A^{2 / 3}, 1.8$ and 3.6, respectively; the solid curve represents $y=g(X)$, the dashed lines $y= \pm A$, and the bold arrow the oscillation amplitude. 
The amplitude of the oscillation decreases monotonically with $\lambda k_{f}$ for $\lambda k_{f} \geqslant \lambda k_{f}^{\mathrm{c}} \equiv 4-3 A^{2 / 3}$. In Figure 5(a) we observe that there are six solutions to eq. (9). On physical grounds, there are two oscillatory motions, one around +1 and the other around -1 , both with the same small amplitude $2 a_{f}$ indicated by arrows along the $x$-axis. As $\lambda k_{f}$ increases, $a_{f}$ also increases slowly and at the critical point $\lambda k_{f}^{\mathrm{c}}=4-3 A^{2 / 3}$ experiences a finite jump; see Figure 5(b). $k_{f}^{\mathrm{c}}$ can be determined from eq. (9) as follows. We define $X^{*}$ (see Figure 5(b)), where the two curves $y=g(X)$ and $y=A$ are tangent to each other. From $\operatorname{dg}(X) /\left.\mathrm{d} X\right|_{X^{*}}=0$, we have

$$
X^{*}=\sqrt{\left(4-\lambda k_{f}^{\mathrm{c}}\right) / 12} \text {. }
$$

Because $g\left(X^{*}\right)=A$, we obtain an important result

$$
\lambda k_{f}^{\mathrm{c}}=4-3 A^{2 / 3}, k_{L}(\lambda, A)=k_{f}^{\mathrm{c}}=\left(4-3 A^{2 / 3}\right) / \lambda,
$$

where $k_{L}$ denotes the degree of the node corresponding to the maximum amplitude $a_{L}$. This result indicates that the maximum amplitude does not always occur on the hub but on the node with degree $k_{L}$. That is, there is a $k_{L}$ for each specific $\lambda$ and the $k_{\text {hub }}$ of the hub is only one of these.

The value of $a_{L}$ is readily determined as follows. From Figure 5(b), $g\left(x^{+}\right)=-A$ and we obtain $X^{+}=A^{1 / 3}$. That is, our unit performs a large amplitude oscillation between $\pm X^{+}= \pm A^{1 / 3}$, with $\pm X^{+}$denoting the non-degenerate solutions to eq. (9) at $\lambda k_{f}=\lambda k_{f}^{\mathrm{c}}$ and this realizes the maximum amplitude

$$
a_{L}=X^{+}=A^{1 / 3}, G=a_{L} / A=A^{-2 / 3} .
$$

If $\lambda k_{f}$ is further increased, $X_{f}$ now starts to decrease from the value in eq. (12).

Because the SF network has a broadly distributed degree, $k$, we can find a node with $k=k_{L}$ for a wide range of $\lambda$. This result is significant because it tells us that the signal response can be amplified for a wide range of coupling strengths $\lambda$, which is necessary and crucial in the application of a signal device. In contrast, for very small $\lambda$, we find no node with degree $k_{L}$, eq. (11), for a finite system and here effects of finite size of the system come into play. This point will be considered later.

To check the validity of eq. (11), we have performed numerical simulations to obtain experimentally $G$ and $k_{L}$, by first constructing 100 different SF networks and then providing each with randomly chosen initial configurations $\left\{x_{i}(t=0)= \pm 1\right\}$ for each $\lambda$. Thus we obtain $100 k_{L}$ for each $\lambda$, from which we calculate the average $\left\langle k_{L}\right\rangle$ and its standard deviation $\delta k_{L}=\sqrt{\left\langle\left(k_{L}-\left\langle k_{L}\right\rangle\right)^{2}\right\rangle}$.

We plot in Figure $6\left\langle k_{L}\right\rangle$ with its standard deviation (the error bar) as a function of the coupling strength $\lambda$; the solid curve is obtained from eq. (11) and the "squares" and "cir- cles" correspond to results for $N=500$ and 2000, respectively. It is easy to see that the theoretical curves agree well with the numerical simulations except in the small $\lambda$ region, confirming our fifty-fifty assumption. We notice also that in the small $\lambda$ region, larger sized $(N=2000)$ networks find better agreement with theory than those of smaller sized $(N=500)$ networks. The reason is that large $N$ has larger $k_{\text {hub }}$ and more links to satisfy the smaller $\lambda$ in eq. (11), i.e. the finite size effect in the small $\lambda$ region. As shown in Figure 6, the experimental results are reproduced by our simple onebody theory rather well.

For a realistic network, an interesting question arises whether the gain changes with coupling strength $\lambda$. Thus we divide the coupling range into three regions: the first region is identified by $0<\lambda<\lambda_{1}$ with $\lambda_{1}$ satisfying $\lambda_{1} k_{\max }=4-3 A^{2 / 3}$, where $k_{\max }$ denotes the largest degree of the concrete network at hand; the second region is $\lambda_{1}<\lambda<\lambda_{2}$ and $\lambda_{2}$ is the point when the system begins to become synchronized; In the third region, $\lambda>\lambda_{2}$, and all the units oscillate with the same phase in the same well $( \pm 1)$ following the input signal $A \sin (\omega t)$. Our theory then predicts that the gain for the second region is given by $G=A^{-2 / 3}$. Thus, in the following, we will mainly focus on the first and third regions.

For the first region, no unit is of sufficient degree $k$ to satisfy eq. (11) for $k_{L}$, which determines the finite size effect seen in Figure 6. Thus, all the units oscillate around their equilibria, i.e. $\approx \pm 1$. For a fixed coupling strength $\lambda$, the maximum amplitude of oscillation is expected to occur on a fifty-fifty unit. Let us set $k_{f}$ equal to $k_{\max }$. From eq. (6) we easily obtain its solution

$$
x_{f}=x_{0}+x_{1}(t)
$$

where $x_{0}= \pm \sqrt{\frac{4-\lambda k_{\max }}{4}}$ is the equilibrium solution of eq.

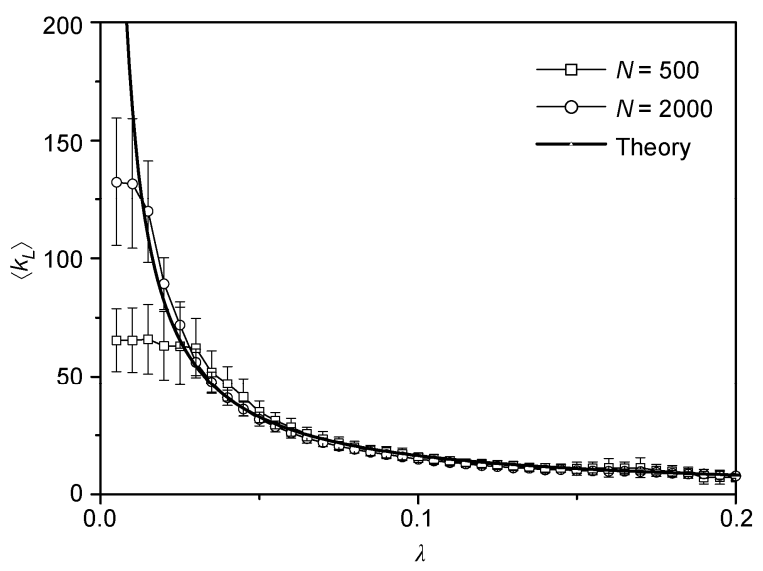

Figure 6 The average $\left\langle k_{L}\right\rangle$ and the standard deviation $\delta k_{L}$ of the degree of the unit $L$ with maximum amplitude are shown, where the "squares" and "circles" represent the cases of $N=500$ and 2000, respectively. The solid curve is obtained from eq. (11). In simulations, we evaluated $100 k_{L}$ for each $\lambda$, which are used to calculate $\left\langle k_{L}\right\rangle$ and $\delta k_{L}$. 
(6) if there is no external force, and $x_{1}(t)$ is a linear response solution

$$
x_{1}=-\frac{A}{4\left(4-\lambda k_{\max }\right)^{2}+\omega^{2}}\left[\omega \cos \omega t-2\left(4-\lambda k_{\max }\right) \sin \omega t\right] .
$$

From $\dot{x}_{f}=0$, we can determine both $X_{\max }$ and $X_{\min }$, and obtain the gain $G \equiv\left(X_{\max }-X_{\text {min }}\right) /(2 A)$ by

$$
G=\frac{1}{\sqrt{4\left(4-\lambda k_{\max }\right)^{2}+\omega^{2}}} .
$$

As $\lambda k_{\max }<4-3 A^{2 / 3}$ in this region, $G$ will be small and slowly increase from $1 / \sqrt{64+\omega^{2}}$ to $1 / \sqrt{36 A^{4 / 3}+\omega^{2}}$ as $\lambda$ increases from 0 to $\lambda_{1}$.

For the third region $\left(\lambda>\lambda_{2}\right)$, the dynamics is fully synchronized, $x_{i}(t)=x(t)(i=1,2, \cdots, N)$. Eq. (2) is reduced to

$$
\dot{x}=-4 x^{3}+4 x+A \sin (\omega t) .
$$

Following the same argument used in deriving eq. (9) from eq. (6), we have from eq. (16)

$$
-4 X^{3}+4 X= \pm A \text {. }
$$

Denoting the solutions for $\pm A$ in eq. (17) by $X_{\max }$ and $X_{\min }$, respectively, we have $a_{\text {synch }}=\left(X_{\max }-X_{\min }\right) / 2$, and thus $G_{\text {synch }}$ $=a_{\text {synch }} / A$ is independent of $\lambda$ in this region.

Thus we obtain the gain for the entire range of coupling strengths. To verify the analysis, we perform numerical simulations on SF networks with $N=500$ and $N=2000$. Figure 7 displays the results for $A=0.4$ ("squares"), $A=0.8$ ("circles") and $A=1.2$ ("triangles"); the solid lines correspond to the theoretical results. From eq. (12), the values for the theoretical gains in the intermediate 'plateau' region are $1.84,1.16$ and 0.88 for $A=0.4,0.8$ and 1.2, respectively. The
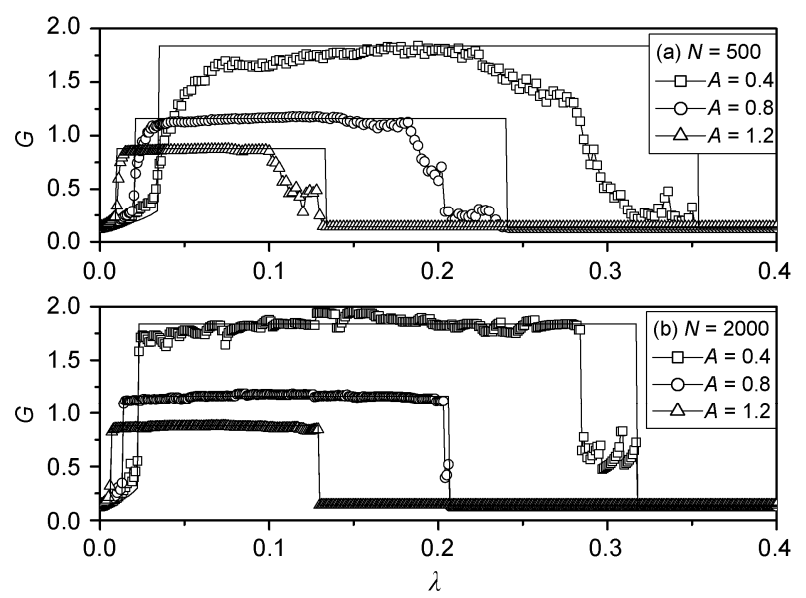

Figure 7 Variation in gain $G$ with coupling strength $\lambda$ for a SF network of size $N=500$ (a) and $N=2000$ (b). The "squares", "circles" and "triangles" represent the signal amplitudes of $A=0.4,0.8$ and 1.2 , respectively, and the lines denote the theoretical results. numerical simulations are in good accord with the theory. In detail, comparing Figure 7(a) with (b), the simulation with $N=2000$ agrees better with the theoretical results than that with $N=500$, confirming that the theoretical results correspond to the large- $N$ limit, $N \rightarrow \infty$. Comparing Figure 7(a) with Figure 2, agreement is only found in the small $\lambda$ region, indicating that the hub is only one of the fifty-fifty units in the one-body theory. Considering that the smallest $\lambda$ determines the sensitivity to weak signals, we say that the hub determines the sensitivity of the system.

\section{Discussions and conclusions}

To begin, the results for signal amplification discussed in this paper are specifically for SF networks. However, in real situations, the configurations of complex networks are defined with different purposes and thus are not limited solely to SF networks. Hence, it would be interesting to study whether signals can be amplified in a general network, i.e. a non-SF network. In this situation, we lose the advantage of the presence of a hub with a large number of links. For the near future, this is a promising but challenging research direction.

A second direction of research in signal amplification is its implementation in a local area of the network. As is well-known, real networks are usually very large. Associated global information is generally unknown and can be estimated only through local measurements $[33,34]$. Thus, it would be useful to amplify local signals, especially those at a specific node. For example, in cell phone networks and ad hoc networks, each user is only concerned with the signal amplification of his/her unit whereas the manager of the network needs to be concerned with those of every node. Furthermore, to deal with all node information in large networks would be very costly in both time and resources, even being sometimes impracticable. Ideally, an approach that amplifies signals using only local information would be interesting to have. We are currently pursuing this direction [35].

A third direction of research could be the implementation of signal amplification with frequency specificity. As mentioned in the introduction, this corresponds to networks having characteristic locations, such as in the cochlea of the inner ear, responding to a specific tone. The field in general is ripe for all manner of research.

In conclusion, we have briefly discussed how the topology of SF networks can be used to amplify signals in artificial information processing systems and devices. Inspiration has been gleaned from the self-tuning signal amplification in auditory systems of animals and insects. Without background noise, both the hub and other nodes in the SF networks have been found to contribute in amplifying the signal response. In the presence of noise, signal amplification was represented by the SNR and a double SR was found. A 
one-body theory was provided to explain the amplified signal response in the context of scale-free networks, where the hub determines the sensitivity of the hearing system. All these results were confirmed by numerical simulations.

This work was partially supported by the National Natural Science Foundation of China (10775052, 10975053, 10635040) and the National Basic Research Program of China (2007CB814800).

1 McCullen N J, Mullin T, Golubitsky M. Sensitive signal detection using a feed-forward oscillator network. Phys Rev Lett, 2007, 98: 254101

2 Camalet S, Duke T, Julicher F, et al. Auditory sensitivity provided by self-tuned critical oscillations of hair cells. Proc Natl Acad Sci USA, 2000, 97: 3183-3188

3 Vilfan A, Duke T. Two adaptation processes in auditory hair cells together can provide an active amplifier. Biophys J, 2003, 85: 191-203

4 Stoop R, Kern A, Gopfert M C, et al. A generalization of the van-der-Pol oscillator underlies active signal amplification in Drosophila hearing. Eur Biophys J, 2006, 35: 511-516

5 Dallos P. The active cochlea. J Neurosci, 1992, 12: 4575-4585

6 Lindner B, Dierkes K, Julicher F. Local exponents of nonlinear compression in periodically driven noisy oscillators. Phys Rev Lett, 2009, 103: 250601

7 Gopfert M C, Robert D. Motion generation by Drosophila mechanosensory neurons. Proc Natl Acad Sci USA, 2003, 100: 5514-5519

8 Martin P, Hudspeth A J. Active hair-bundle movements can amplify a hair cell's response to oscillatory mechanical stimuli. Proc Natl Acad Sci USA, 1999, 96: 14306-14311

9 Ospeck M, Eguiluz V, Magnasco M. Evidence of a Hopf bifurcation in frog hair cells. Biophys J, 2001, 80: 2597-2607

10 Seo B, Krishnan R, Munakata T. Self-tuning of threshold for a two-state system. Phys Rev E, 2007, 75: 056106

11 Lindner J F, Meadows B K, Ditto W L, et al. Array enhanced stochastic resonance and spatiotemporal synchronization. Phys Rev Lett, 1995, 75: 3-6

12 Liu Z, Lai Y C, Nachman A. Enhancement of noisy signals by stochastic resonance. Phys Lett A, 2002, 297: 75-80

13 Liu Z, Lai Y C, Nachman A. Enhancement of detectability of noisy signals by stochastic resonance in arrays. Int $\mathrm{J}$ Bif Chaos, 2004, 14: 1655-1670

14 Albert R, Barabasi A L. Statistical mechanics of complex networks. Rev Mod Phys, 2002, 74: 47-97

15 Boccaletti S, Latora V, Moreno Y, et al. Complex networks: Struc- ture and dynamics. Phys Rep, 2006, 424: 175-308

16 Sporns O, Chialvo D R, Kaiser M, et al. Organization, development and function of complex brain networks. Trends Cogn Sci, 2004, 8: 418-425

17 Eguiluz V M, Chialvo D R, Cecchi G A. Scale-free brain functional networks. Phys Rev Lett, 2005, 94: 018102

18 Zhou C, Zemanova L, Zamora G, et al. Hierarchical organization unveiled by functional connectivity in complex brain networks. Phys Rev Lett, 2006, 97: 238103

19 Palmer J. Brain works more like internet than 'top down' company. http://www.bbc.co.uk/news/science-environment-10925841

20 Acebron J A, Lozano S, Arenas A. Amplified signal response in scale-free networks by collaborative signaling. Phys Rev Lett, 2007, 99: 128701

21 Liu Z, Munakata T. Scale-free topology-induced double resonance in networked two-state systems. Phys Rev E, 2008, 78: 046111

22 Lu F, Liu Z. Frequency sensitivity of signal detection in scale-free networks. Chin Phys Lett, 2009, 26: 040503

23 Kondo T, Liu Z, Munakata T. One-body theory for amplified signal response in a scale-free network. Phys Rev E, 2010, 81: 041115

24 Barabasi A L, Albert R. Emergence of scaling in random networks. Science, 1999, 286: 509-512

25 Liu Z, Lai Y C, Ye N, et al. Connectivity distribution and attack tolerance of general networks with both preferential and random attachments. Phys Lett A, 2002, 303: 337-344

26 Benzi R, Sutera A, Vulpiani A. The mechanism of stochastic resonance. J Phys A, 1981, 14: L453-458

27 McNamara B, Wiesenfeld K. Theory of stochastic resonance. Phys Rev A, 1989, 39: 4854-4869

28 Longtin A. Stochastic resonance in neuron models. J Stat Phys, 1993, 70: 309-327

29 Gammaitoni L, Hanggi P, Jung P. Stochastic resonance. Rev Mod Phys, 1998, 70: 223-287

30 Russell D F, Wilkens L A, Moss F. Use of behavioural stochastic resonance by paddle fish for feeding. Nature, 1999, 402: 291-294

31 Lindner B, Garcia-Ojalvo J, Neiman A, et al. Effects of noise in excitable systems. Phys Rep, 2004, 392: 321-424

32 Zaikin A A, Kurths J, Schimansky-Geier L. Doubly stochastic resonance. Phys Rev Lett, 2000, 85: 227-231

33 Liang X, Liu Z, Li B. Weak signal transmission in complex networks and its application in detecting connectivity. Phys Rev E, 2009, 80: 046102

34 Wang Z, Chen Q, Liu L, et al. Relationship between topology and functions in metabolic network evolution. Chinese Sci Bull, 2009, 54: 776-782

35 Zhou J, Zhou Y, Liu Z. Amplification of signal response at an arbitrary node of a complex network. Phys Rev E, 2011, 83: 046107

Open Access This article is distributed under the terms of the Creative Commons Attribution License which permits any use, distribution, and reproduction in any medium, provided the original author(s) and source are credited. 